\title{
Relating Web pages to enable information-gathering tasks
}

\author{
Amitabha Bagchi \\ Dept of Computer Science \& Engg \\ Indian Institute of Technology \\ New Delhi 110016
}

\author{
Garima Lahoti \\ Cazoodle Inc. \\ 60 Hazelwood Drive, Suite 122, \\ Champaign, IL 61820-7460
}

December 2, 2018

\begin{abstract}
We argue that relationships between Web pages are functions of the user's intent. We identify a class of Web tasks - information-gathering - that can be facilitated by a search engine that provides links to pages which are related to the page the user is currently viewing. We define three kinds of intentional relationships that correspond to whether the user is a) seeking sources of information, b) reading pages which provide information, or c) surfing through pages as part of an extended information-gathering process. We show that these three relationships can be productively mined using a combination of textual and link information and provide three scoring mechanisms that correspond to them: SeekRel, FactRel and SurfRel. These scoring mechanisms incorporate both textual and link information. We build a set of capacitated subnetworks - each corresponding to a particular keyword - that mirror the interconnection structure of the World Wide Web. The scores are computed by computing flows on these subnetworks. The capacities of the links are derived from the hub and authority values of the nodes they connect, following the work of Kleinberg (1998) on assigning authority to pages in hyperlinked environments. We evaluated our scoring mechanism by running experiments on four data sets taken from the Web. We present user evaluations of the relevance of the top results returned by our scoring mechanisms and compare those to the top results returned by Google's Similar Pages feature, and the Companion algorithm proposed by Dean and Henzinger (1999).
\end{abstract}

\section{Introduction}

The tremendous success of collaborative filtering-based recommendation systems (see e.g. [20]) in online retail settings (e.g. Netflix) has demonstrated that users welcome guidance while looking for books to buy or films to rent i.e. where they are not looking for a product which satisfies a general specification rather than a specific product. In the enterprise search space, the increasing importance of faceted search - essentially a method of providing recommendations to satisfy a user's search needs by creating multiple taxonomies - pioneered by Endeca [12, under the name "guided navigation", shows that businesses are recognizing that they can improve profitability by effectively helping their employees and customers browse through large databases by providing search results related to the ones users express a preference for.

But search engines for the World Wide Web have been largely unsuccessful in providing accurate and helpful recommendations to their users. Research has found that most Web users are not using advanced features provided by search engines; it has been shown that they barely understand what these features do [29]. Further, it has been seen that Web users search less and browse more [2]. And, in fact, the process of developing expertise in using the Web coincides with an increase in browsing and decrease in searching [8]. 
Despite this bleak scenario it is our contention that search engines have the resources to effectively provide users with related pages that can help in information-gathering tasks. Further we believe that a search engine which can do this will increase its value for its users, with consequent increases in revenue. There is a growing understanding in the search domain that user intent is crucial to the search process [27, 15]. Extending this understanding to the domain of relationships between pages can make information-gathering tasks easier.

In other words, to know the relationship between two pages, we must first know what function these two pages serve for the users who visit them. It is only by characterizing the task the user is engaged in that we can offer related pages and hope that these will actually facilitate the task. In this paper we provide a suite of scoring mechanisms that relate Web pages: SeekRel, FactRel and SurfRel. The purpose of these scoring mechanisms is to help identify pages which may be related to the current page depending on whether the user is reading pages which provide information, seeking links to sources of information, or surfing through pages as part of an extended information-gathering process.

A brief overview of our method is as follows: We compute our scores using flow calculations in a set of subnetworks of the Web. Each subnetwork corresponds to a single keyword. The set of subnetworks used to score a pair of pages is decided by finding the keywords relevant to the pair of pages being scored. Then the edges of these networks are capacitated using the hub values [18] of their originating pages. Finally flow is sent along these edges towards special nodes we call witnesses. The amount of flow that can be routed is used as a measure of the relationship.

Organization. In Section 2 we focus on information-gathering tasks and identify how our recommendations can facilitate them. We survey previous work done in relating Web pages in Section 3 , Our specific scoring mechanisms are described in Sections 4.1 and 4.2. A comparison of our results for a small toy network with the results produced by two recent proposals PageSim [21] and SimRank [16] is presented in Section 4.3. In Section 5 we present experiments conducted on real data taken from the Web. We discuss the results of user surveys that compared the top results produced by our scoring mechanisms with the results given by the Companion algorithm of Dean and Henzinger [10] and Google's Similar Pages feature. Finally in Section 6 , we conclude by discussing the merits of our scheme in comparison to these two proposals and by arguing that our scheme is a better candidate for inclusion in a search engine than the Companion algorithm.

\section{Information-gathering on the Web}

The tasks that Web users undertake were classified by Broder [6] to generally fall into three categories: navigational (finding specific pages), informational (seeking facts) and transactional (performing some interactive set of tasks.) Kellar [17] further classifies informational tasks into information-seeking, information exchange and information maintenance tasks. It is in the first of these classes, information seeking, that search engines make their major contributions. Kellar differentiates between three types of information seeking tasks: fact-finding e.g. directions to a friend's house or exam dates, informationgathering e.g. tectonic movements, Mac laptops, browsing news, friend's homepage. See Figure 1 for a schematic representation of Kellar's classification (Source [17.)

Our focus is on information-gathering which we distinguish from fact finding by using Kellar's definition: Information-Gathering consists of tasks in which a user is collecting information, often from multiple sources, in order to write a report, make a decision, or become more informed about a particular topic [17, pp 67]. With this definition in hand let us try to characterize this class of tasks. 


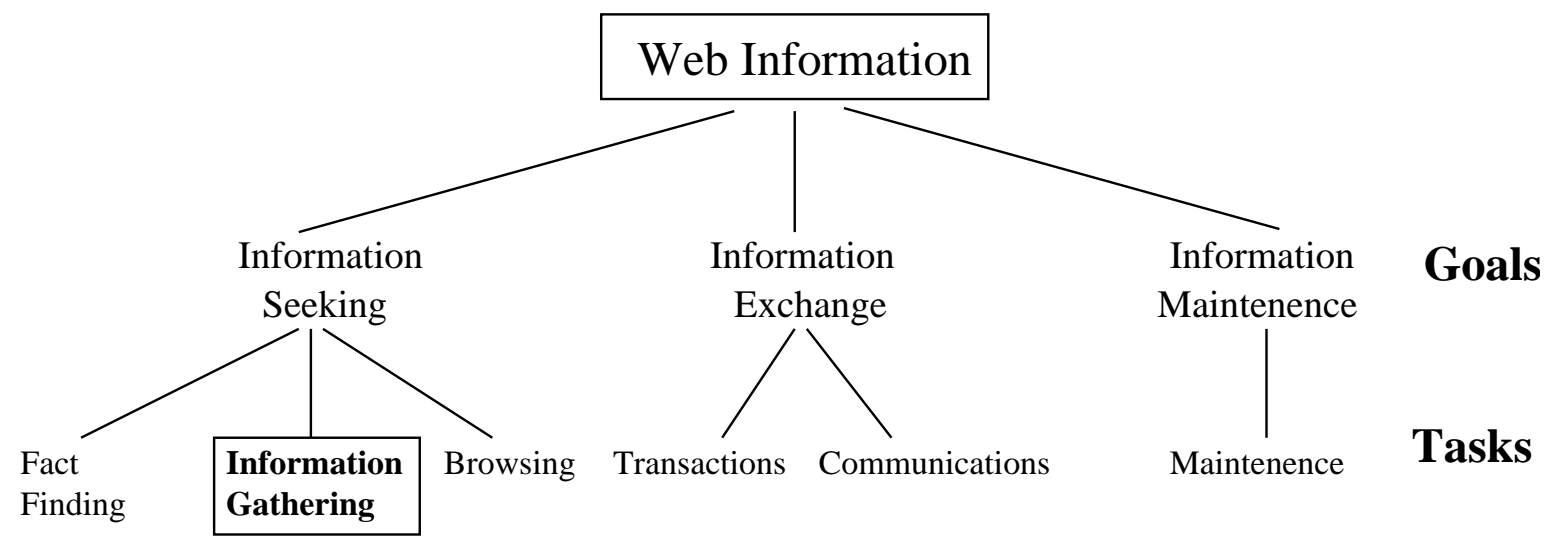

Figure 1: Kellar's classification of Web information. Source: Kellar, 2007.

\subsection{The iterative nature of information-gathering tasks}

In their seminal work Belkin, Oddy and Brooks [3, 4, pointed out that assuming that a user with a need for information will be able to specify that need exactly is a mistake. Instead, they pointed out that a user comes to an information retrieval system with an anomalous state of knowledge, which she then attempts to express as a query. Based on the information received the anomaly is rectified to some extent, the user's image of the world is altered somewhat. But this is not the end of the process. The altered state of the user's knowledge generates new anomalies which she then takes back to the information retrieval engine in the form of requests or queries. This iterative process continues till the user is satisfied with the extent of the change in his state of knowledge.

Belkin et. al.'s work has been refined in several directions (we refer the reader to Marchioni's book for a survey [24]) but the general view remains a powerful organizing principle for information retrieval. This general view is borne out in the World Wide Web setting by Rose's conclusion [27] - based on earlier studies he and Levinson conducted [28] - that information search was an iterative process. Other supporting evidence in this regard is Aula et. al.'s finding that experienced Web user's showed a pattern of searching, then browsing, the searching again [2], which was similar to Cothey's finding in a longitudinal study that followed subjects evolving from novice to expert [8].

With all of this as background we characterize the information gathering task on the World Wide Web as an iterative one (see Figure 2). This task is initiated by an understanding that the user's knowledge needs to be augmented, proceeds by looking for Web pages (either from search engines or other sources) and then browsing them when they are found. The process of browsing rectifies the knowledge anomaly partially or wholly and has the additional effect of providing links to other pages which might aid the process. The user may then choose to follow those other links (immediately or later) or finish browsing the current page. When a page is browsed completely the user is either satisfied entirely and terminates the task, or is partially satisfied and resumes the task by again looking for Web pages.

This iterative characterizations of the information-gathering tasks suggests ways in which search engines could aid users in performing them. It is to this that we now turn our attention.

\subsection{Enabling information-gathering tasks}

In Figure 2 we notice that a user browsing a Web page in the process of gathering information treats the page either as a source of information or as a source of links to other pages. It is therefore appropriate to provide users with links to two kinds of pages: 


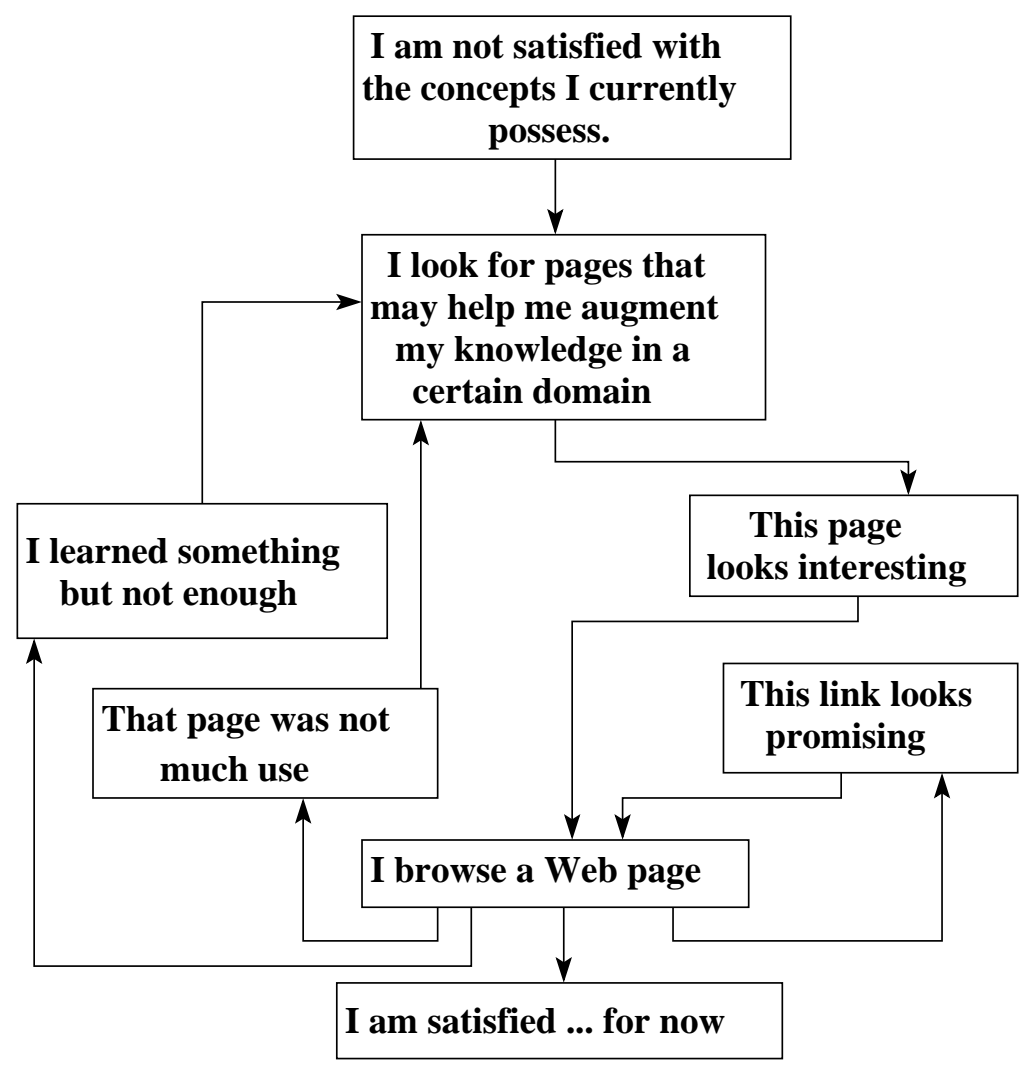

Figure 2: A schematic of information-gathering on the Web. 
P1. Pages which contain information similar to the current page.

P2. Pages which provide links similar to the links provided by the current page.

Additionally it is our contention that as user experience with the Web improves, there will be the realization that people who create Web content and Web links have an understanding of the interrelationships between various pages. And so we suggest that a third kind of page could be useful in the information-gathering process:

P3. Pages which can be reached by following a sequence of links from the current page and pages from which the current page can be reached by following a sequence of links.

The specifications $\mathbf{P 1}$ and $\mathbf{P 2}$ are general and open to interpretation as to how they might be satisfied. The pages of $\mathbf{P 3}$ are more specific but there is still the question of which one of these pages to choose to present to the user from among the several thousand that might satisfy this criterion. In Section 4 we will provide three scoring mechanisms: FactRel will account for pages of type P1, SeekRel for P2 and SurfRel for P3.

How should these links be provided to the user? While this is a question better addressed by experts in human-computer interaction, we would suggest that the "toolbars" provided by many search engines could be augmented to provide page recommendations. These already provide information about the "rank" of the page currently being viewed and various other pieces of information from the search engine's bag, and this additional use can fit in seamlessly. For searches that take place on a search engine's Web page, each "similar pages" link can contain these related pages. In either case it is of paramount importance that the links provided be appropriately categorized so that the user can choose to follow them (or not) depending on the particular function the page being viewed (or the initial search result) plays in her information-gathering process.

\section{Related work}

The relationship between Web pages has been studied fairly extensively. Most researchers use the term "similarity" and define it variously. In this paper we have consciously avoided the use of this term since we believe that the relationship between Web pages is not intrinsic to the pages but depends on the functionality of the pages for the user. However, in order to survey previous contributions to this area, in this section we will use the term "similarity."

There are two broad categories of approaches to the Web page similarity problem. The first relies mainly on the textual content of Web pages. The pair of pages being compared is either seen just as two groups of items (keywords, anchor text, patterns in the text) which overlap significantly or as structured entities that resemble each other in their organization e.g. which tags appear next to which ones. Since this is not our approach we do not review this vast literature here, referring the reader to [30] for a succinct survey.

The second approach involves taking link and interconnection information into account. An important way in which interconnection has been used is to ascribe authority to a page based on which pages link to it. This idea forms the basis of the Pagerank algorithm employed by Google [5]. In a similar vein, Kleinberg [18] described two attributes of Web pages: they can be authorities on a topic, or they can be hubs, linking to pages which are authoritative. He described an iterative algorithm to compute measures of these two attributes for each Web page. One line along which Kleinberg's work was developed involved using anchor text as a descriptive summary of the page being linked to [7, 13]. But, more relevant to our methods was the focus on the link structure seen in Dean and Henzinger's paper [10] where they gave two algorithms for finding similar Web pages. One of their algorithms used 
the idea of co-citation earlier seen in Pitkow and Pirolli's work [26]. The second algorithm, called Companion shares an important feature with our scoring mechanisms: it uses Kleinberg's algorithm. Their method of building a focused graph extends Kleinberg's ideas. We have used their ideas for building capacitated subnetworks in our algorithm. While Dean and Henzinger give a list of pages similar to a given page, Huang et. al. 14 gave measures of similarity based on the predecessor and successor set, and also on the basis of all the vertices reachable from the two pages. Another sort of "closure" of co-citation was used to define a similarity measure called PageSim in [21]. In this measure, pages propagate their similarity measure to their neighbors, the importance of a particular propagation being decided by the PageRank of the page. This measure was an improvement on the SimRank measure proposed in [16] which worked on the principle that two pages are similar if they are linked to by similar pages. The SimRank measure was shown to be specific instance of a general framework for computing similarity between heterogeneous data objects by Xi et. al. [31] who proposed the SimFusion algorithm. In Section 4.3 we will compare the results PageSim and SimRank produce on a small toy example with the results given by our scoring mechanisms.

In a different use of link structure related to our own Lu et. al. 23, 22, claimed that two pages were said to be similar if flow could be routed from one of them to the other. However, unlike our work, their capacity assignments were not based on any notion of authority. To the best of our knowledge this is the only other mention of using flow to score similarity in the literature.

As mentioned, the literature on finding similarity is vast and has seen contributions from many different areas. The papers we have discussed above are the ones whose techniques are closely related to our own. With these in view we now proceed to describing our scoring mechanisms.

\section{Computing relationship scores}

In this section we describe our algorithms for scoring the relationships between a pair of pages $u$ and $v$. It is our contention that two pages should have a high FactRel score if it is possible to find paths from multiple pages to both of them. We say that a page $z$ witnesses the FactRel relationship between $u$ and $v$ if it is possible to reach both $u$ and $v$ from $z$ by following a series of links. For example, in Figure 3, the page $G$ witnesses FactRel between $A$ and $B$. Similarly we say that a page $z$ witnesses the SeekRel relationship if it is possible to reach $z$ by following a series of links from $u$ to $z$ and $v$ to $z$. For example, in Figure 3, $C$ witnesses SeekRel between $G$ and $I . F$ is another such witness for these two. Somewhat differently, the SurfRel relationship does not require explicit witnesses. We say that the SurfRel relationship exists between $u$ and $v$ if it is possible to reach $u$ from $v$ by following a series of links, or vice versa. In Figure 3, we see that $H$ and $B$ are related this way, while $A$ and $I$, are not related by SurfRel.

Hence our scores are based on finding paths in the World Wide Web. But which paths should be given more weight than others in the scoring process? To answer this question we rely on Kleinberg's notion [18] of authorities (pages which have credible information) and hubs (pages which link to good authorities) in order to derive a relevant set of focused capacitated subnetworks from the structure of the World Wide Web. We then determine relationship scores by sending flow to the two pages from witnesses (for FactRel), from the two pages to witnesses (for SeekRel) or between the pages (for SurfRel) in these capacitated networks.

We will now discuss these methods in detail. In Section 4.1 we will describe how to build our set of capacitated subnetworks using Kleinberg's method for assigning authority to Web pages. In Section 4.2 we describe how to find witnesses, route flow and compute scores. We illustrate the working of the algorithm on a toy example in Section 4.3 and also present a comparison by scoring Simrank [16] and PageSim [21] on the same example. 


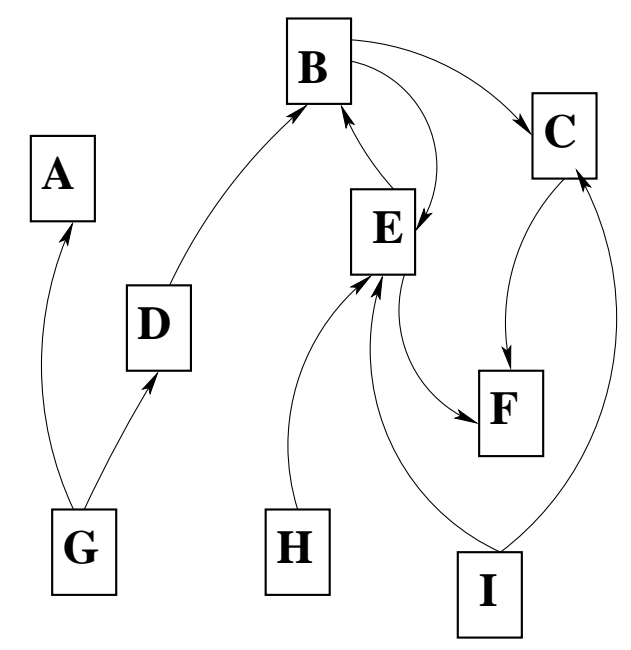

Figure 3: A simple hyperlinked network

\subsection{Building capacitated subnetworks}

We begin by assuming that we have a database of significant keywords, call it $\mathcal{K}$. We also assume that there is some function $\gamma: \mathcal{K} \rightarrow(0,1)$ which assigns relative importance to these keywords. We do not specify how to build $K$, preferring to rely on the wealth of tools available for this purpose (see, e.g. [32]). For each of these keywords we apply Kleinberg's [18. HITS algorithm to compute hub and authority values for all the pages associated with that keyword. Finally, at the end of this preprocessing step each page has hub and authority values computed for the subset of the database of keywords it is associated with. When we are given a pair of pages to score we identify sets of significant keywords $K_{u}$ and $K_{v}$ for $u$ and $v$. We then merge these two sets to get the top $k$ keywords for some tunable parameter $k$. This set of chosen keywords we call $K$. Once we have this set of keywords we proceed by making a set of networks $\mathcal{N}=\left\{N_{w} \mid w \in K\right\}$. For each $N_{w}$ we first find the set of Web pages $P_{w}$ which contains the keyword $w$.

Since we will be using Kleinberg's hub and authority values [18] to capacitate the network (in Section 4.2) we grow $N_{w}$ by first taking all the pages that link to the pages in $P_{w}$ and the pages that are linked to by the pages in $P_{w}$ (as described in [18]). Then we add the refinements to this structure proposed by Dean and Henzinger [10. Only the main feature of these refinements has been mentioned in Steps 2c and 2d, The reader is referred to [10] for further details.

Finally, for each network $N_{w}$ we run Kleinberg's algorithm for assigning hub and authority values to each page and label a node $z$ of network $N_{w}$ with these values: $\operatorname{hub}_{w}(z)$ and $\operatorname{auth}_{w}(z)$. A directed edge from node $x$ to node $y$ is assigned capacity $\operatorname{hub}_{w}(x)$.

A summary of the algorithm is in Figure 4. We postpone a discussion of the motivation for this construction to Section 4.3 .

\subsection{Using flows to compute relationship scores}

Finding witnesses. For both SeekRel and FactRel we have to find witnesses in each $N_{w}$. In Figure 5 we describe a simple algorithm that uses breadth-first search from both $u$ and $v$ upto $d$ levels for some value of $d$ to return a sorted list, $S_{w}$, of witnesses for SeekRel. Note that we do not just create a set of witnesses, but actually make an ordered list of witnesses. The significance of this will become clear shortly. In order to construct a list of witnesses for FactRel we simply reverse the direction of all the 


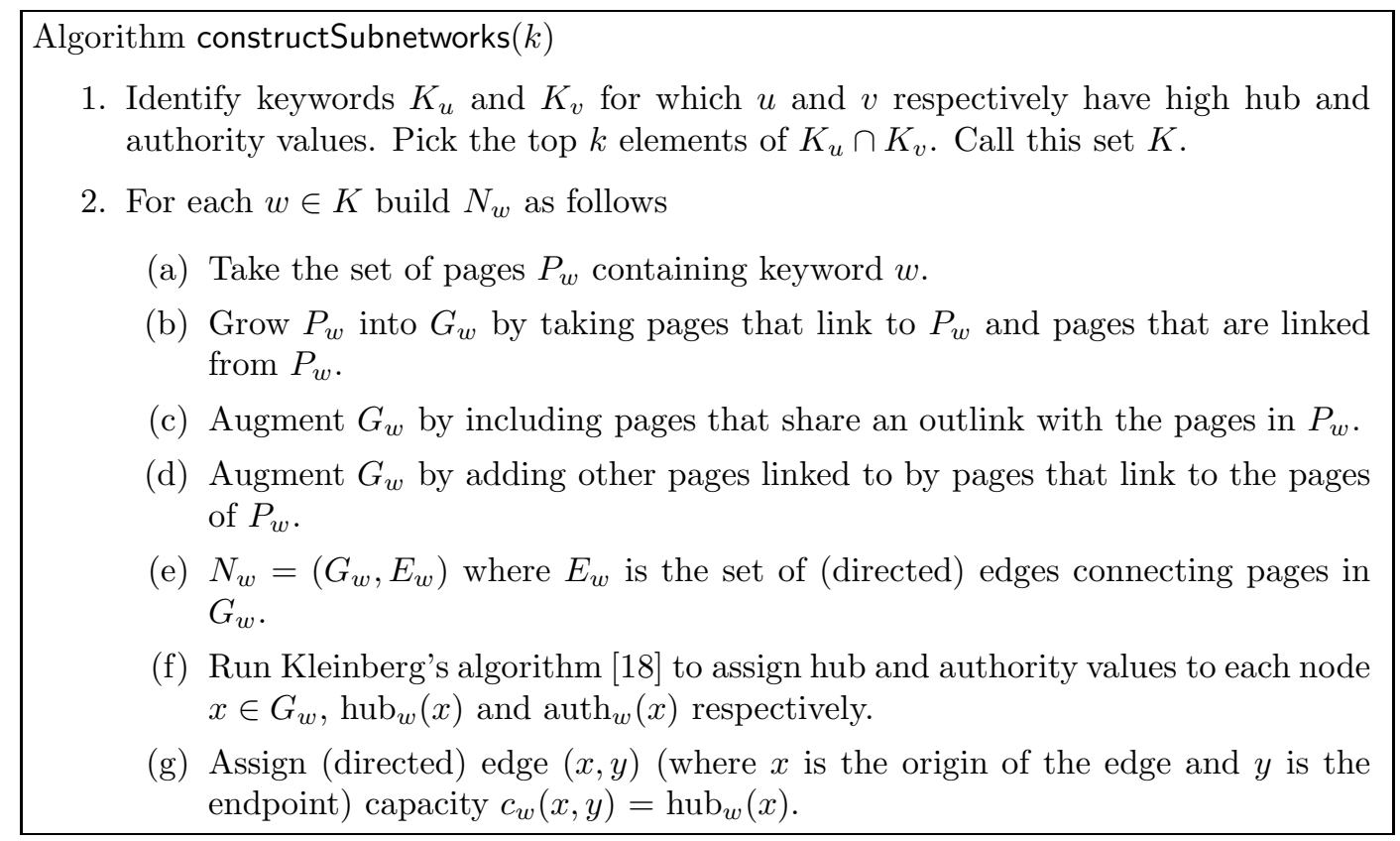

Figure 4: Building a set of capacitated subnetworks

edges of $N_{w}$ and execute exactly the same algorithm. We denote the set of witnesses for FactRel by $F_{w}(u, v)$. Note that the reversal of edges is only to find witnesses, not to compute flows, that takes place on the same graph for both SeekRel and FactRel.

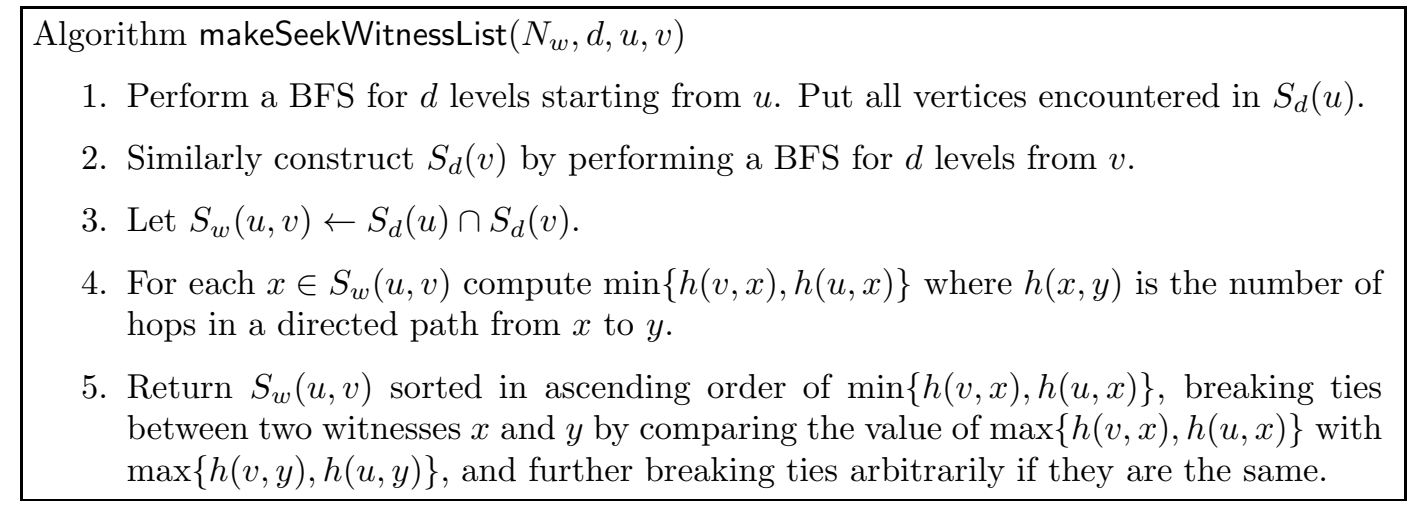

Figure 5: Finding SeekRel witnesses for $u$ and $v$.

Computing flows. Having constructed the ordered list of witnesses we go down the list one vertex at a time using any standard single-source maximum flow algorithm for computing the max flow first from $u$ to or from the witness as required, then from $v$. Note that when computing the flow from $u$ to a witness we eliminate $v$ from the network and vice versa. This is to ensure that the one page doesn't piggyback on the other in order to route flow to a witness i.e. all the flow $v$ sends to a witness is independent of $u$ and vice versa. After computing the flow for a particular witness, we reduce the capacity of the edges into or out of the witness. Let us postpone discussing the rationale and method for this to the end of this section. We then move on to the next witness in the list. See Figure 6 for a formal description of the algorithm for computing flows for SeekRel. The algorithm for computing 
flows for FactRel is symmetric. In this case we denote the flow from a witness $x$ as factFlow ${ }_{w}(x)$.

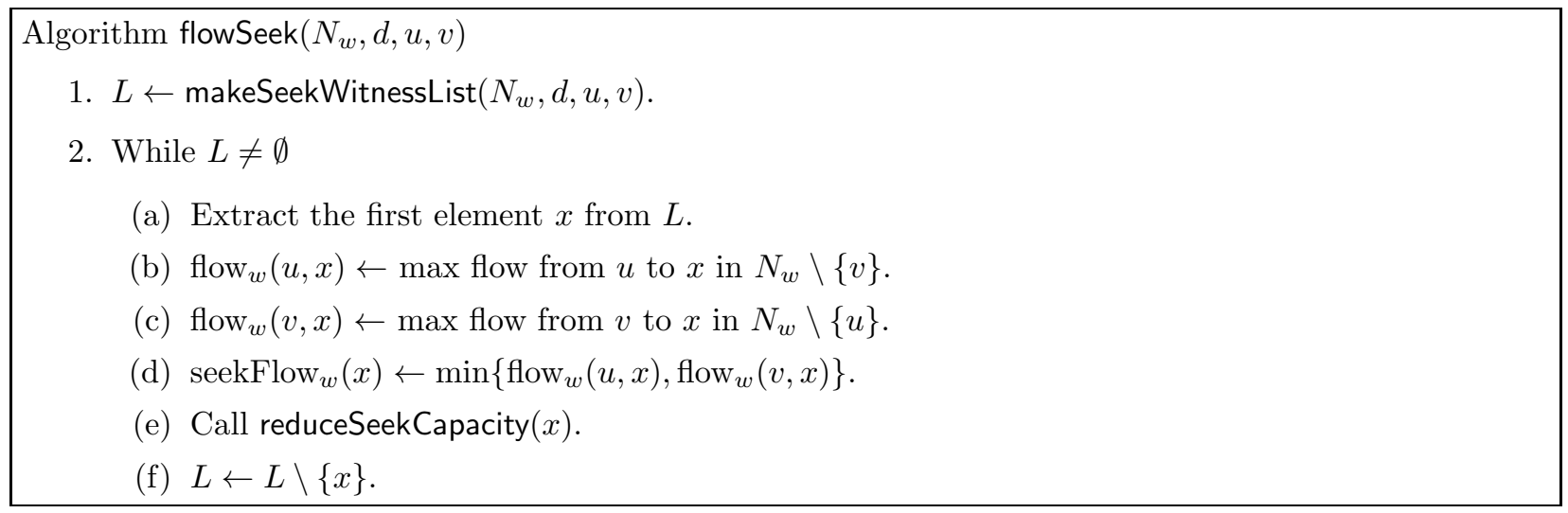

Figure 6: Finding the witness flow for SeekRel

The scores. In order to compute the relationship scores we have to be able to combine flows from different subnetworks. In order to do this we normalize all flows by dividing by the weight of the maximum edge in the network, a quantity we denote by maxwt $(w)$. Also, we factor in the relative importance of the various keywords in $K$ using the function $\gamma$. Hence our first two scores are

$$
\begin{aligned}
& \operatorname{SeekRel}(u, v)=\sum_{w \in K} \frac{\gamma(w)}{\operatorname{maxwt}(w)} \cdot\left(\sum_{x \in S_{w}(u, v)} \operatorname{seekFlow}_{w}(x)\right) \cdot \\
& \operatorname{FactRel}(u, v)=\sum_{w \in K} \frac{\gamma(w)}{\operatorname{maxwt}(w)} \cdot\left(\sum_{x \in F_{w}(u, v)} \operatorname{factFlow~}_{w}(x)\right) .
\end{aligned}
$$

The rationale behind FactRel is that pages providing similar information will be identified by witnesses in the network, which act collaboratively to identify good sources of information and link to them. Similarly, the intuition between SeekRel is that two pages which allow a user to reach more or less the same pages are related in terms of their ability to guide the user as she navigates the Web.

The third measure, SurfRel is easier to compute since it requires no witnesses. We simply compute the max flow from $u$ to $v$ in $N_{w}$, denoting it flow $w(u \rightarrow v)$ and the flow from $v$ to $u$, denoted flow $w \rightarrow$ $u)$. Now, we can say that

$$
\operatorname{SurfRel}(u \rightarrow v)=\sum_{w \in K} \frac{\gamma(w)}{\operatorname{maxwt}(w)} \cdot \text { flow }_{w}(u \rightarrow v) .
$$

SurfRel encapsulates the idea that if the Web allows one page to reach another through a simple sequence of clicks, these two pages must be related because they are both likely to be visited in a single browsing session. This idea of two pages being related by the presence of a path between them is very intuitive and the concept of flow generalizes the idea of paths. By capacitating edges with the hub value of the nodes they originate at, we differentiate between nodes and their ability to allow information to propagate by looking at their credibility as hubs in the hyperlinked environment. The more the credibility of the node as a hub, the more the flow it can forward.

Reducing witness capacity. In the case of SeekRel and FactRel, the presence of many witnesses that can sink or source a lot of flow from the two pages being scored (or send a lot of flow to them) leads 
to a higher score for the pair. But there are cases where this score may be artificially high. Consider the network in Figure 7. $E, B, C$ and $G$ all witness SeekRel for $H$ and $I$. But the flow to $B, C$ and $G$ all goes through $E$. So these three are redundant, in the sense that the information they provide is already contained in the fact that $E$ is a witness for $H$ and $I$.

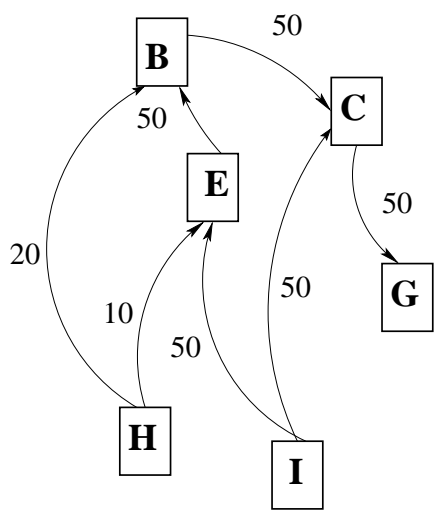

Figure 7: Redundant witnesses. Capacities are marked on edges.

It is to prevent these redundant witnesses from artificially inflating the relationship score that we reduce the capacity associated with the witness in Step 2e of the flow computing algorithm of Figure 6 , For SeekRel when we are done computing flow to a witness we reduce its incoming capacity before moving on to the next witness in the list. For FactRel the outgoing capacity is reduced. Before we describe the algorithm formally in Figure 8 let us define some notation. For a vertex $x$ let the set of incoming edges be $I(x)$ and the set of outgoing edges be $O(x)$. Let the flow routed for vertex $u$ on edge $e$ be $f_{u}(e)$. The capacity of edge $e$ is $c(e)$.

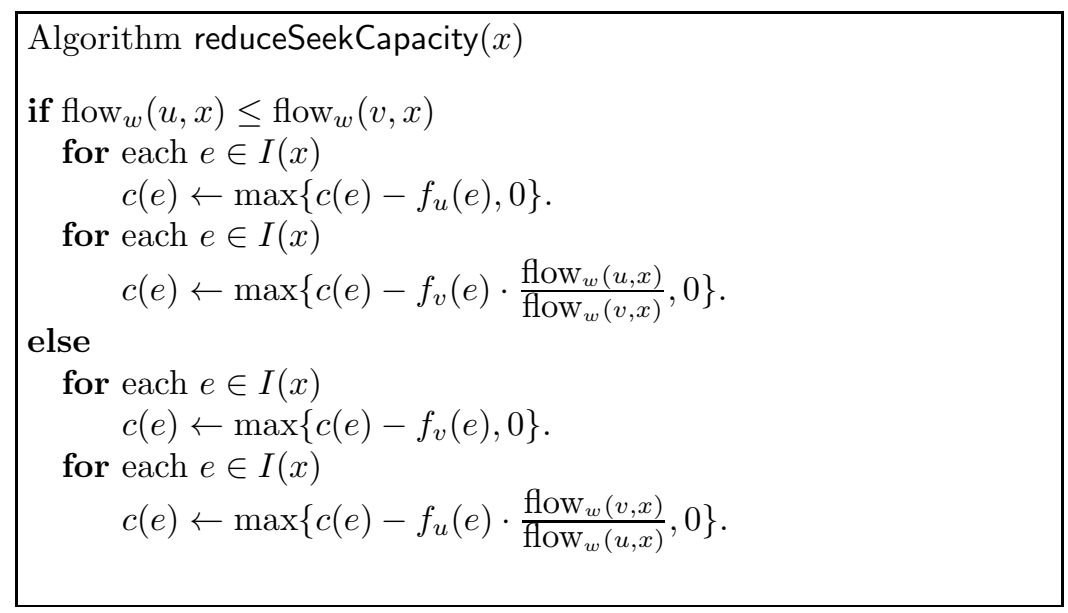

Figure 8: Reducing capacities for SeekRel

Essentially what reduceSeekCapacity $(x)$ does is remove the amount of flow witnessed at $x$. Since we take the minimum of $\operatorname{flow}_{w}(u, x)$ and $\operatorname{flow}_{w}(v, x)$ as the amount of flow being witnessed, we remove this amount from the incoming capacity of $x$. And to ensure we do this fairly for both $u$ and $v$, we penalize the incoming edges used by both the flows flow $_{w}(u, x)$ and flow $w(v, x)$ equally by scaling down the larger flow to the smaller one before subtracting it from the capacity of the incoming edge. 
The algorithm reduceFlowCapacity $(x)$ is symmetric to this, only it removes capacity from the outgoing edges of the witness. In both these cases, with the witness capacity reduced, the ability of redundant witnesses to skew the flow reduces.

The reason for fixing an ordering for our witnesses becomes clear now since the capacities of the network decrease after each flow calculation. Clearly the order in which the witnesses are processed will make a difference to the flow that is routed to them. Recall the function $h(u, v)$ defined in Figure 5 as the number of hops in a directed path from $u$ to $v$. Let us see what the values of $(h(x, v), h(x, u))$ are for $v=H$ and $u=I$. For $G$ this is $(3,2)$, for $C$ it is $(2,1)$, for $B$ it is $(1,2)$ and for $E$ it is $(1,1)$. So the sorted order according to our algorithm should be $E, B, C, G$. For this the total flow witnessed will be 10 units for $E$ plus 20 units for $B=30$ units. Because of capacity reduction $C$ and $G$ will not be able to witness any flow. But is this correct given that $I$ has a direct edge to $C$ which doesn't go through $B$ or $E$ ? We argue it is since the flow of 30 that could be witnessed at $C$ is the same as the flow of 30 units that is being witnessed for $H$ at $B$ and $E$. In Figure 7 for example, if we choose the witnesses in the reverse order $G, C, B, E$ the total flow is 30 witnessed by $E+30$ witnessed by $C+$ 30 witnessed by $B+10$ witnessed by $E$, totalling 100 , of which 70 units are redundant.

Thus it follows that distant witnesses along a chain of nodes are more likely to be redundant since decreasing their capacity will not affect the flow to nearer witnesses. With this in mind, and also noting that more information about a Web page is likely to be found in its near neighborhood rather than far away from it, we order witnesses in increasing order of the distance of the witness from one of the pages in (Step 5 of algorithm makeSeekWitnessList $\left(N_{w}, d, u, v\right)$ in Figure 5. If a distant witness still witnesses flow after the reduction of capacity of nearer witnesses, we can be sure that this is not redundant flow.

\subsection{Discussion}

We took the simple subnetwork of Figure 9 and ran our scoring algorithms on it. The table of scores obtained is in Figure 10. For cleanness of presentation all hub values have been scaled by 1000. The flow values have been scaled up by maxwt $=815$ since we are only considering one subnetwork.

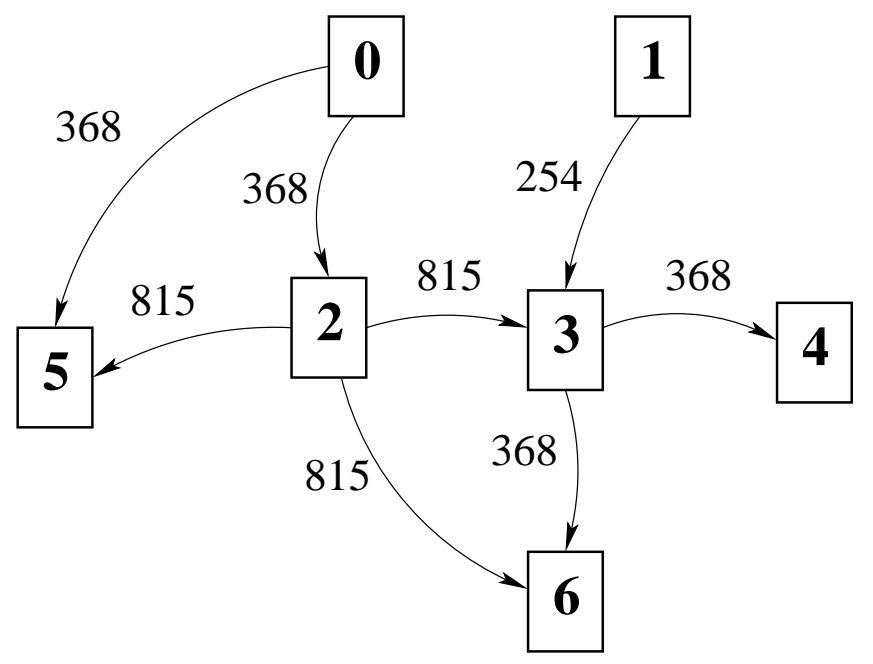

Figure 9: A simple subnetwork with edge capacities according to the hub values of the originating node.

In Figure 9 we see that node 2 is related by SeekRel to nodes 0 and 3. This is as it should be since they both point to similar parts of the network. Node 0 sends 368 units of flow to the witness 


\begin{tabular}{|c||c|c|c|c|c|c|c|}
\hline & 0 & 1 & 2 & 3 & 4 & 5 & 6 \\
\hline \hline 0 & & $(253,0,0,0)$ & $(368,0,368,0)$ & $(368,0,368,0)$ & $(0,0,368,0)$ & $(0,0,736,0)$ & $(0,0,368,0)$ \\
\hline 1 & $(253,0,0,0)$ & & $(253,0,0,0)$ & $(0,0,253,0)$ & $(0,0,253,0)$ & $(0,0,0,0)$ & $(0,0,253,0)$ \\
\hline 2 & $(368,0,0,368)$ & $(253,0,0,0)$ & & $(368,0,815,0)$ & $(0,0,368,0)$ & $(0,368,815,0)$ & $(0,0,1183,0)$ \\
\hline 3 & $(368,0,0,368)$ & $(0,0,0,253)$ & $(368,0,0,815)$ & & $(0,0,368,0)$ & $(0,815,0,0)$ & $(0,815,368,0)$ \\
\hline 4 & $(0,0,0,368)$ & $(0,0,0,253)$ & $(0,0,0,368)$ & $(0,0,0,368)$ & & $(0,736,0,0)$ & $(0,368,0,0)$ \\
\hline 5 & $(0,0,0,736)$ & $(0,0,0,0)$ & $(0,368,0,815)$ & $(0,815,0,0)$ & $(0,736,0,0)$ & & $(0,1183,0,0)$ \\
\hline 6 & $(0,0,0,368)$ & $(0,0,0,253)$ & $(0,0,0,1183)$ & $(0,815,0,368)$ & $(0,368,0,0)$ & $(0,1183,0,0)$ & \\
\hline \multicolumn{8}{|c|}{ Legend: (SeekRel $(x, y)$, FactRel $(x, y), \operatorname{SurfRel}(x \rightarrow y), \operatorname{SurfRel}(y \rightarrow x))$}
\end{tabular}

Figure 10: Relationship scores for the network of Figure 9 (scaled up by $1000 \times$ maxwt)

\begin{tabular}{|c||c|c|c|c|}
\hline & SeekRel & FactRel & SurfRel $\rightarrow$ & SurfRel $\leftarrow$ \\
\hline \hline 0 & 2,3 & None & 5 & None \\
\hline 1 & 0,2 & None & $3,4,6$ & None \\
\hline 2 & 0,3 & 5 & 6 & 0 \\
\hline 3 & 0,2 & 5,6 & 4,6 & 2 \\
\hline 4 & None & 5 & None & $0,2,3$ \\
\hline 5 & None & 6 & None & 2 \\
\hline 6 & None & 5 & None & 2 \\
\hline
\end{tabular}

Figure 11: High scorers for Figure 9

5 which is matched by 2 . Node 3 sends 368 units of flow to witness 6 , which is also matched by 2 . And although the node 1 shares many witnesses with 0 , the flow it can send is limited by its outgoing capacity (which is low because it is not a good hub) and so its SeekRel score is low, though non-zero, and 2 and 3 beat it out in scoring.

If we look at the second column of the table in Figure 11, we see that 5 and 6 are the top scorers for FactRel for several nodes. Node 2's high credibility as a hub helps draw attention to 5 and 6 . The node 0's good hub value helps relate 2 to 5 , in what can seen to be an example of pure co-citation.

In the case of SurfRel, the example of 0 is interesting. While 0 is strongly related to 5 by this measure, its score with 6 is relatively less. In this case our score captures the fact that there are multiple independent paths from 0 to 5 while all paths from 0 to 6 go through 2 . The divergence in the paths after 2 does not help boost SurfRel for 0 and 6 .

To further illustrate the power of our methods, we implemented the SimRank [16] and PageSim [21] scoring algorithms and scored our simple subnetwork using them. The results are in Figures 12 and 13 ,

\begin{tabular}{|c||c|c|c|c|c|c|c||c|}
\hline & 0 & 1 & 2 & 3 & 4 & 5 & 6 & High Score \\
\hline \hline 0 & 1.0 & 0.0 & 0.0 & 0.0 & 0.0 & 0.0 & 0.0 & None \\
\hline 1 & 0.0 & 1.0 & 0.0 & 0.0 & 0.0 & 0.0 & 0.0 & None \\
\hline 2 & 0.0 & 0.0 & 1.0 & 0.0 & 0.0 & 0.5 & 0.0 & 5 \\
\hline 3 & 0.0 & 0.0 & 0.0 & 1.0 & 0.0 & 0.25 & 0.25 & 5,6 \\
\hline 4 & 0.0 & 0.0 & 0.0 & 0.0 & 1.0 & 0.0 & 0.5 & 6 \\
\hline 5 & 0.0 & 0.0 & 0.5 & 0.25 & 0.0 & 1.0 & 0.25 & 2 \\
\hline 6 & 0.0 & 0.0 & 0.0 & 0.25 & 0.5 & 0.25 & 1.0 & 4 \\
\hline
\end{tabular}

Figure 12: SimRank scores for Figure 9,

SimRank related none of the pages to either 0 or 1 whereas our SeekRel is able to detect the fact that 0 can aid in helping the user find links to pages that 2 and 3 can also lead to. Even 1 shares this property as a navigational aid with some of the other pages, a fact that comes up in our scoring. 
The case of node 1 is particularly interesting because PageSim, that gives non-zero scores in many cases where SimRank fails, does not deduce 1's relationship to 0 and 2 that our SeekRel is able to find. A user currently viewing 1 would come to believe that only 3 and 4 are related to 1 if she relied on PageSim or SimRank. This would be erroneous because a knowledge that 0 is related in terms of links it provides could lead that user to 2 , which she would not find if she relied on these other two measures. PageSim is somewhat more sophisticated than SimRank so it detects 0's relationship to 5, just like our SurfRel $\rightarrow$ does, but it misses 0's relationship to 3 that we find through SeekRel.

\begin{tabular}{|c||c|c|c|c|c|c|c||c|}
\hline & 0 & 1 & 2 & 3 & 4 & 5 & 6 & High Score \\
\hline 0 & 0.08 & 0.0 & 0.04 & 0.01 & 0.01 & 0.05 & 0.02 & 5 \\
\hline 1 & 0.0 & 0.08 & 0.0 & 0.08 & 0.04 & 0.0 & 0.04 & 3 \\
\hline 2 & 0.04 & 0.0 & 0.16 & 0.05 & 0.03 & 0.08 & 0.08 & 5,6 \\
\hline 3 & 0.01 & 0.08 & 0.05 & 0.33 & 0.16 & 0.05 & 0.19 & 6 \\
\hline 4 & 0.01 & 0.04 & 0.03 & 0.16 & 0.33 & 0.03 & 0.16 & 3,6 \\
\hline 5 & 0.05 & 0.0 & 0.08 & 0.05 & 0.03 & 0.25 & 0.06 & 2 \\
\hline 6 & 0.02 & 0.04 & 0.08 & 0.19 & 0.16 & 0.06 & 0.42 & 3 \\
\hline
\end{tabular}

Figure 13: PageSim scores for Figure 9 ,

PageSim almost misses 5's relationship to 4 and also scores 5's relationship to 6 quite low. SimRank completely misses the relationship to 4 and scores the relationship to 6 lower than the relationship to 2. On the other hand, a high FactRel score for both of these allows a user to tell that the information available at 4 and 6 are both relevant to people who are interested in 5. Since our FactRel score between 5 and 2 is relatively lower and our SurfRel score between them is high, a user can deduce the nature of the relationship between 5 and 2, a fact also detected by SimRank.

We now move on to experiments on real data taken from the Web.

\section{Experimental evaluation}

\subsection{Experimental setup}

We performed our experiments on four data sets taken from the Web. Creating these data sets was a multi-stage process that began by querying AltaVista [1] with a search string and taking the top 100 results to form a core set. We did not use Google since we compare our results to Google's Similar Pages feature. We then used the open source Web crawler Nutch [25] to retrieve the pages linked from the core set. Then we found the top 1000 pages that link to these new pages using Altavista's advanced feature providing inlinks for a queried page. Finally, we found the inlinks of the pages in the core using Altavista then went back to Nutch to find the outlinks of these pages. We followed Dean and Henzinger [10] and took only the top 10 outlinks in the manner they specified i.e. if we were looking at the outlinks of a page $u$ which pointed to a core page $v$, we took only the links on $u$ which were "around" the link to $v$ in the sense that we took the 5 links immediately preceding the link to $v$ on the page and the 5 links immediately following $v$. Having obtained this data set we preprocessed it by computing the hub and authority values of all the pages in it.

Our four data sets were generated using the keyword strings "automobile" (54952 pages), "motor company" (14973 pages), "clothes shopping" (37724 pages) and "guess" (12101 urls). For repeatability purposes, these data sets have been made available online 1 We conducted extensive experiments on all these data sets by taking one page out of them as a query, then scoring all three relationships for this

\footnotetext{
${ }^{1}$ http://www.cse.iitd.ernet.in/ bagchi/relationship-scores/
} 
page with all the other pages in the data set. Note that this does not exactly correspond to our claim in Section 4 that we proceed by extracting keywords from pairs of pages and then computing flows on the subnetworks obtained from those keywords. Limitations on the amount of data we were equipped to handle in a university setting prevented us from performing these steps in the full. We present here these stripped down experiments as indicative of what a full implementation of our scoring mechanisms might be able to achieve.

We compared our 10 top scoring pages for FactRel and SeekRel with the top 10 pages returned by Google's Similar Pages feature. We also implemented the Companion algorithm described in [10] and compared our results to the top 10 results returned by it. For SurfRel we simply took our top 10 results and evaluated them. The evaluation in all these cases was done by conducting user surveys.

For each of the target URLs scored using FactRel we asked the user to imagine they had visted it in the course of an information-gathering task and found it relevant. We then assembled a set of 30 URLs (FactRel's top 10, Google's top 10 and Companion's top 10). We presented these 30 URLs in a random order and asked users to answer three yes/no questions: 1) Would you visit this page if you had already visited the target page? 2) Does this page provide similar information to the target page? and 3) Is this page relevant to your information-gathering task?

Each such survey was given to between 5 and 8 users. For each of the 30 pages, and each of these 3 questions a relevance score was computed. For a given target URL $t$, and a result page $i$ :

$$
\operatorname{Rel}(t, i)=\frac{\text { Number of YES answers }}{\text { Number of users who took the survey }}
$$

For each target page and each algorithm, the Precision at $r$ of the ranked results was computed using the formula

$$
\text { Precision-at- } r(t, r)=\frac{\sum_{i=1}^{r} \operatorname{Rel}(i)}{r} .
$$

The precision at $r$ for an algorithm was computed by taking the average of the precision at $r$ values over all the target pages evaluated.

For SeekRel the first and the third question remained the same. The second question was replaced with Does this page provide links similar to those in the target page? Precision at $r$ was calculated similarly for SeekRel. For SurfRel we only asked one question: Is this page relevant to your task? and we did not present results from Google or Companion.

The code for all scoring mechanisms was written in Java (JDK-1.5.0). The open source Nutch crawler was downloaded and run and a parser was written in $\mathrm{C}++$ to parse its output. A total of 9 target pages were evaluated for FactRel, 7 for SeekRel and 5 for SurfRel. On the 14973 page "motor company" data set finding all three relationship scores between a given query page and all the other query pages on a desktop PC with a $3.4 \mathrm{GHz}$ Intel Pentium processor with 1GB RAM took about 8 minutes on average for this data set. On the 37,724 page "clothes shopping" data set it took about 1 hour on average to calculate all three scores of a given page with all other pages. Let us now see what the experiments revealed.

\subsection{Experimental results}

In Figure 14 we list the 10 URLs that scored the highest on FactRel for the page www.honda.com. The relationship revealed is expected: other major car companies. More interesting is a list of pages related by FactRel to www.cngvehicle.com. Not only do we get pages related to other alternate fuels (Biodiesel Forum (forums.biodiesel.com), Electric Drive Transportation Association (evaa.org) and government agencies dealing with renewable energy policy, we also get links to private car industry players who are pursuing the development of energy efficient cars. 


\begin{tabular}{|c||c||c|}
\hline & www.honda.com & www.cngvehicle.com \\
\hline \hline 1 & www.ford.com & www.evaa.org \\
\hline 2 & www.toyota.com & forums.biodieselnow.com \\
\hline 3 & www.landrover.com & www.eere.energy.gov/cleancities \\
\hline 4 & www.audi.com & www.ford.com \\
\hline 5 & www.gm.com & www.nrel.gov \\
\hline 6 & www.mercuryvehicles.com & www.eere.energy.gov/cleancities/- \\
\hline 7 & www.cadillac.com & www.gsa.gov/Portal/gsa/ep/- \\
\hline 8 & www.chevrolet.com & www.mercuryvehicles.com \\
\hline 9 & www.lincoln.com & www.gm.com \\
\hline 10 & www.porsche.com & www.honda.com \\
\hline
\end{tabular}

Figure 14: FactRel top scorers for two pages of the "motor company" data set.

\begin{tabular}{|c||c||c|}
\hline & FactRel & Google's "similar pages" \\
\hline \hline 1 & www.macys.com & www.saksfifthavenue.com \\
\hline 2 & www.neimanmarcus.com & www.macys.com \\
\hline 3 & www.jcpenney.com & www.nordstrom.com \\
\hline 4 & www.abercrombie.com & www.neimanmarcus.com \\
\hline 5 & www.bananarepublic.com & www.barneys.com \\
\hline 6 & www.bluefly.com & www.fds.com \\
\hline 7 & www.spiegel.com & www.starbucks.com \\
\hline 8 & www.saksfifthavenue.com & www.walmart.com \\
\hline 9 & www.target.com & www.nycvisit.com \\
\hline 10 & www.eddiebauer.com & www.ritzcarlton.com \\
\hline
\end{tabular}

Figure 15: FactRel vs Google's "similar pages" for www.bloomingdales.com

For the Web page of the clothing store Bloomingdales 2 , FactRel and Google's "similar pages" returned more or less identical lists right at the top, but FactRel's high scorers remained very focused (other apparel stores) while Google provided links to coffee shops and hotels which could possibly be appropriate in some contexts but deviate from what is arguably the main focus of a user visiting the Bloomingdale's Web page (see Figure 15).

As another demonstration of FactRel's reliability in providing alternate sources of information, we present its top scorers for www.mysimon.com, a comparison shopping site, in Figure 16, FactRel scored Web pages for major comparison shopping sites very high.

To test the robustness of FactRel we scored the home page of Guess Jeans (www.guess.com) using not just the "clothes shopping" data set but also the "guess" data set. Despite the presence of the ambiguous keyword "guess", FactRel's top 10 results were closely related to the original page: pages relating to clothing and accessories. Google's similar pages, on the other hand, appeared to get severely misled by the keyword "guess" (see Figure 17). The precision at $r$ graph for www.guess.com in Figure 18 reveals that Google does very poorly while FactRel and Companion provide good results.

In general we found that FactRel's results were substantially better than Google's but not better than those returned by Companion. In Figure 19 we see that users preferred to visit the top 10 pages presented by FactRel over those of Google after having visited the target page.

For SeekRel the picture was more complex. While users generally felt that the pages presented by SeekRel were far better than the results presented by Google and Companion in terms of the similarity

${ }^{2}$ www.bloomingdales.com 


\begin{tabular}{|c||c|}
\hline & FactRel for www.mysimon.com \\
\hline \hline 1 & www.dealtime.com \\
\hline 2 & shopping.yahoo.com \\
\hline 3 & www.ebay.com \\
\hline 4 & www.bizrate.com \\
\hline 5 & www.pricegrabber.com \\
\hline 6 & www.nextag.com \\
\hline 7 & www.become.com \\
\hline 8 & www.alibris.com \\
\hline 9 & www.buy.com \\
\hline 10 & www.www.bestbuy.com \\
\hline
\end{tabular}

Figure 16: FactRel high scorers for www.mysimon.com

\begin{tabular}{|c||c||c|}
\hline & FactRel & Google's "similar pages" \\
\hline \hline 1 & www.gap.com & www.guessthename.com \\
\hline 2 & www.gucci.com & www.onlineshoes.com $/ \ldots$ \\
\hline 3 & www.marciano.com & www.sonypictures.com $/ \ldots$ \\
\hline 4 & www.guessinc.com & www.imdb.com $/$ title $/$ tt0372237 \\
\hline 5 & www.jcrew.com & www.amazon.com $/ \mathrm{Guess} / \ldots$ \\
\hline 6 & www.gbyguess.com & www.bizrate.com $/ .$. guess +bags.html \\
\hline 7 & www.hugoboss.com & www.learner.org/... \\
\hline 8 & www.givenchy.com & popular.ebay.com/...Guess+Jeans.html \\
\hline 9 & www.gianfrancoferre.com & www.answers.com/topic/guess-inc \\
\hline 10 & www.diesel.com & www.guessfinancial.com \\
\hline
\end{tabular}

Figure 17: FactRel vs Google's "similar pages" for www.guess.com

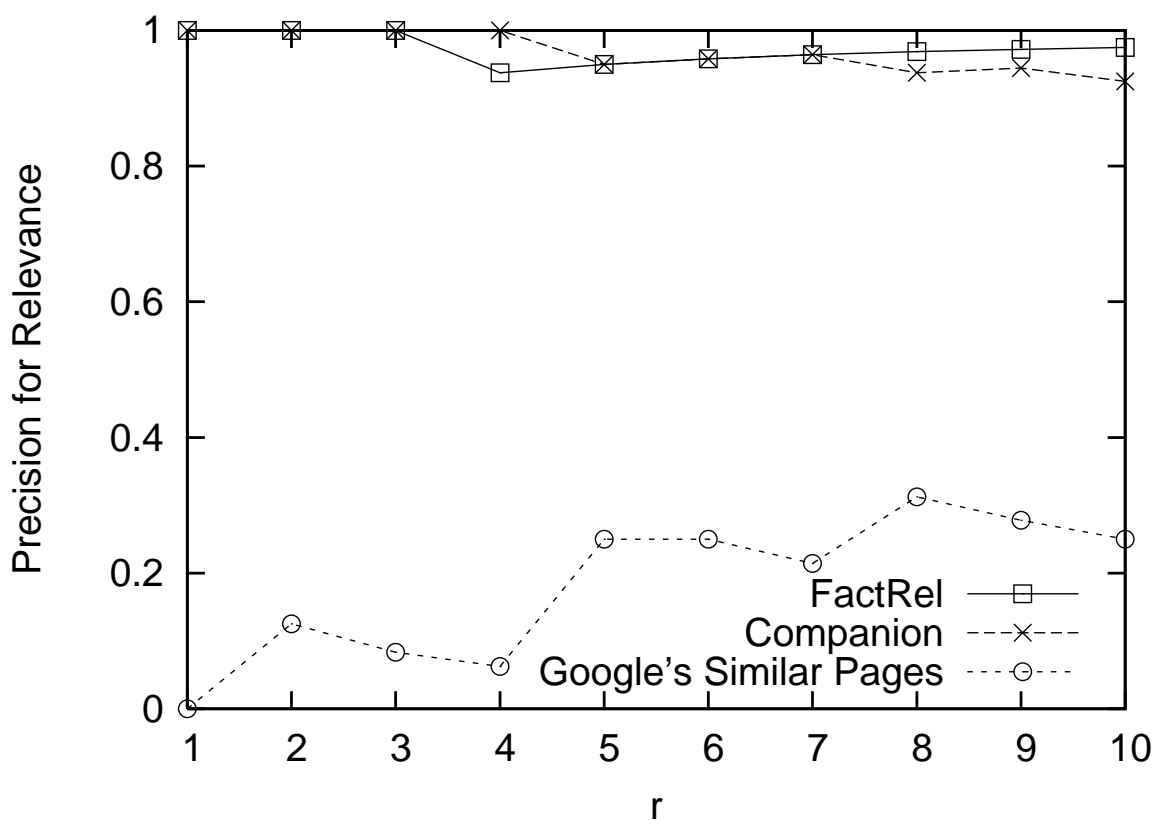

Figure 18: Precision at $r$ for the relevance question for www.guess.com. 


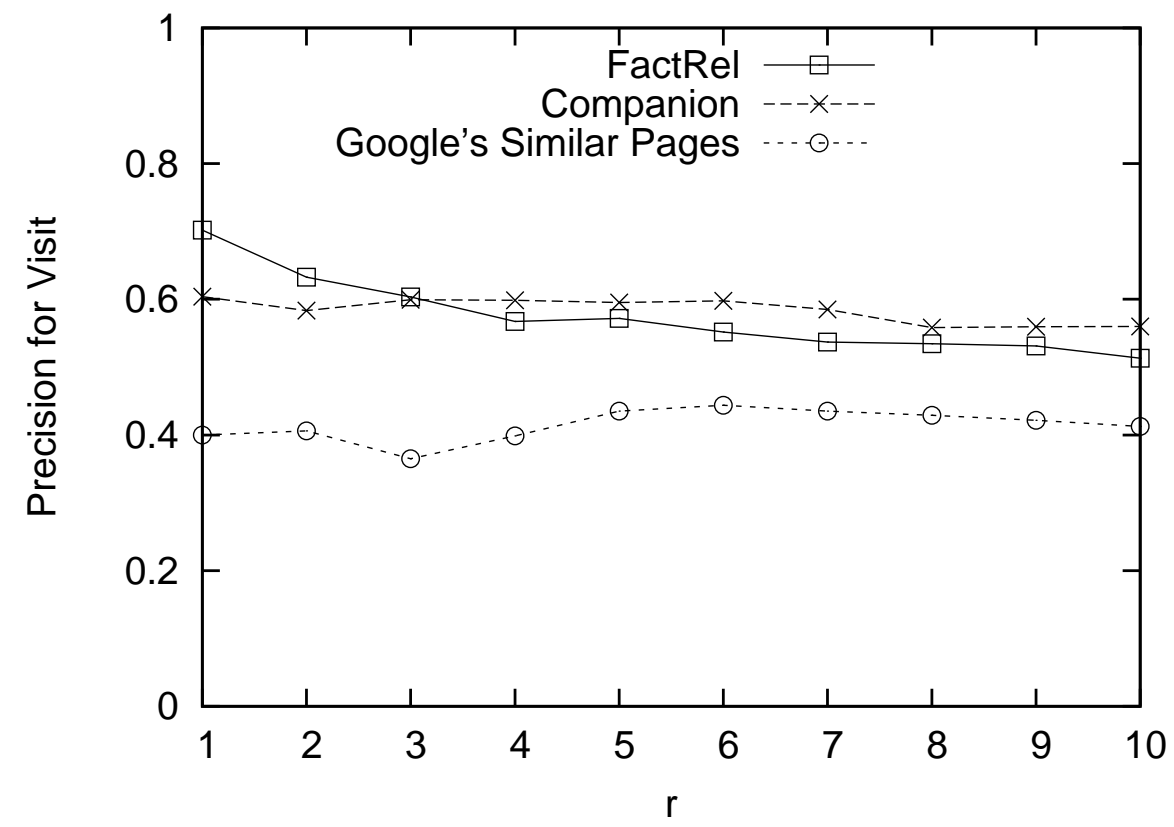

Figure 19: Precision at $r$ for the visit question for the FactRel target pages.

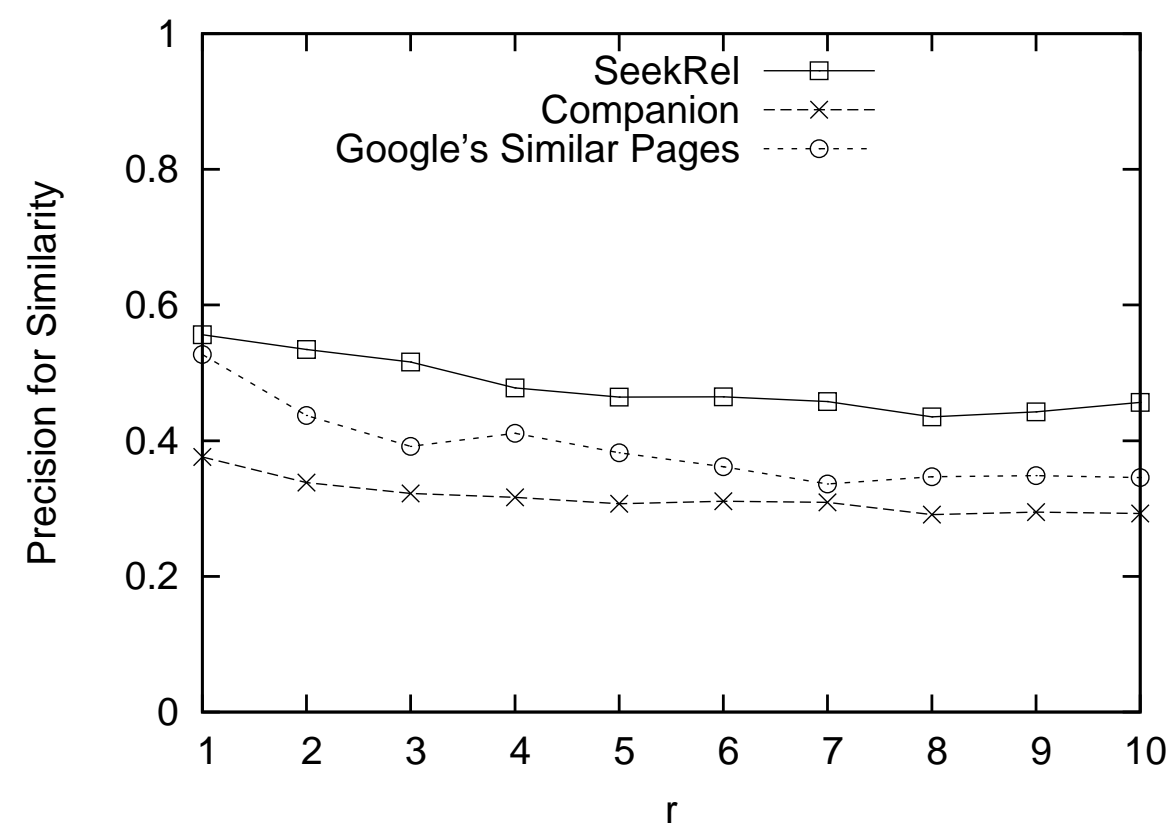

Figure 20: Precision at $r$ for the similar links question for the SeekRel target pages. 


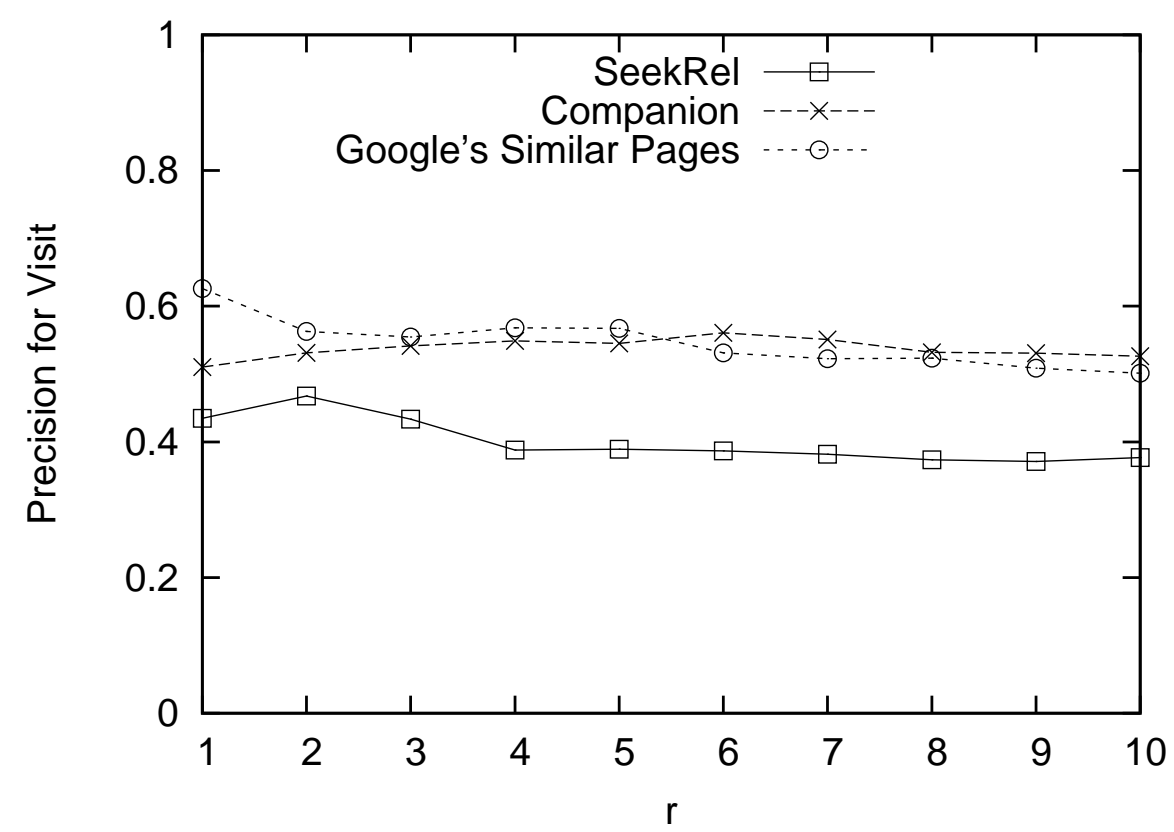

Figure 21: Precision at $r$ for the visit question for the SeekRel target pages.

\begin{tabular}{|c|}
\hline www.shoppingcolumn.com/personal-shopping.html \\
\hline www.misslist.com/stores/shoes.html \\
\hline www.arlingtoncards.com/aroundtown/bizshop1.htm \\
\hline www.allwi.com/wipresents.html \\
\hline www.digital-librarian.com/shopping.html \\
\hline www.cool1055.com/lc/features/shopping \\
\hline www.ersys.com/usa/13/1349000/mall.htm \\
\hline
\end{tabular}

Figure 22: SeekRel top scorers for ersys.com's mall information page for Santa Clara, CA.

of the links on them to those on the target page (see Figure 20), they preferred to vist the pages presented by the other two algorithms (see Figure 21). This is strong independent evidence in favour of Aula et. al.'s conclusion [2] that Web users prefer to browse rather than search. Rather than visit another page with links similar to a given page they would rather visit a page with actual information on it.

Another possible drawback in SeekRel was revealed when we scored for a page listing all the malls in the Santa Clara, California area 3 (see Figure 22). We found that pages with links to online shopping resources and even personal shopping options scored high. But we also found that pages with local information for places as far afield from Santa Clara as Macon, Georgia appeared near the top of the list. The absence of geographical domain knowledge in our system shows up here.

The user response to SurfRel was fairly good. Almost half of our top ten results were found relevant by the respondents (see Figure 23).

\footnotetext{
${ }^{3}$ www.ersys.com/usa/06/0669084/mall.htm.
} 


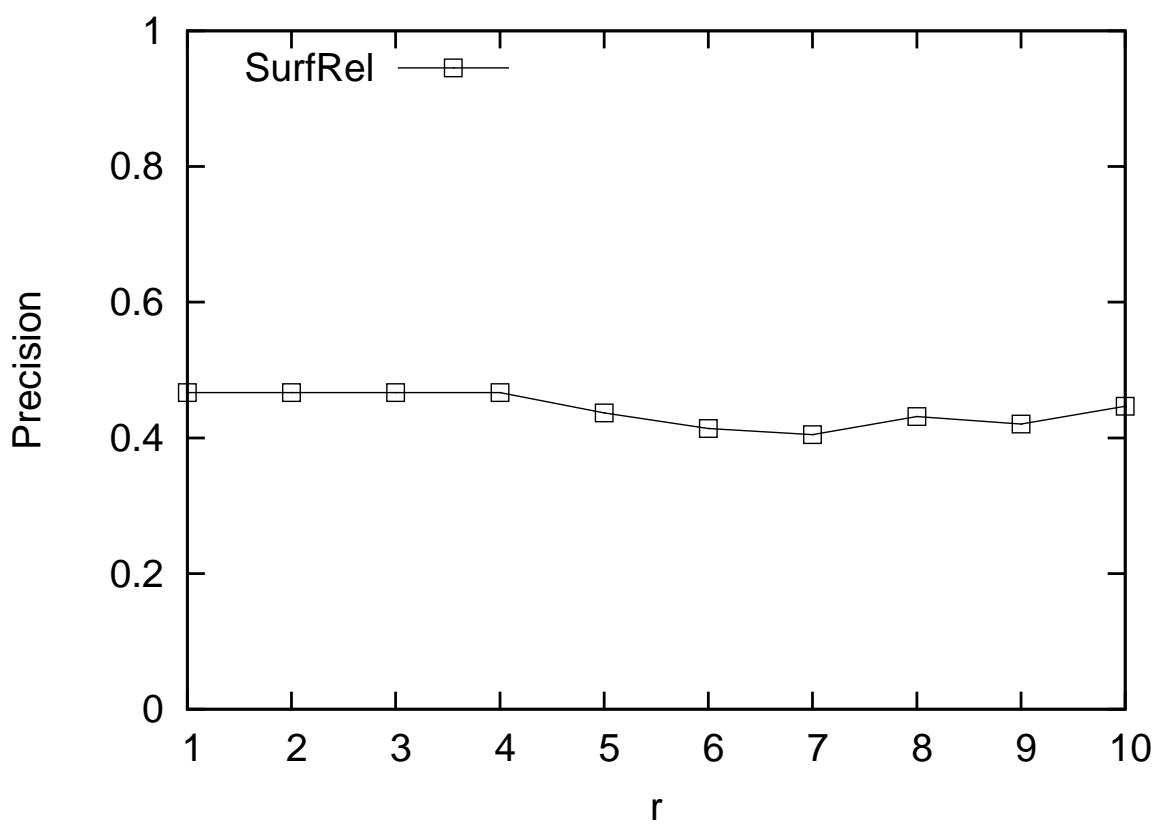

Figure 23: Precision at $r$ for SurfRel.

\section{Discussion}

Our scoring mechanisms are designed with a view to integrate the two broad streams of thought on web page relationships: textual and link-based. This goes some way in addressing Lawrence and Giles' criticism that search engines are biased towards pages which are well-linked [19] and is hence an advantage over algorithms like Dean and Henzinger's Companion which take only link information into consideration.

Although users ranked our results in the same ballpark as Companion, it is our contention that our algorithms are much less resource intensive and much more suited to inclusion in a real-world search engine. We maintain a deck of subnetworks, one corresponding to each significant keyword. It is difficult to estimate the number of significant keywords but if we take the widely used lexical database WordNet [11] as an indicator, the number is of the order of 100,000 4 The Companion algorithm, on the other hand, creates a subnetwork for each queried page. Creating the subnetwork at query time is difficult because of the overhead involved in crawling the Web (or even an image of the web stored on disk) and preprocessing and storing these networks appears infeasible given that the size of the World Wide Web is estimated to be in the tens of billions of pages [9]. Even if it were feasible to store structural information on such a scale, the problem of updation is hard to solve. The Web is constantly changing, and updating our keyword-based subnetworks will be an order of magnitude less resource intensive than updating billions of page-specific subnetworks.

Our approach is further vindicated by the observation that the results provided by FactRel and SeekRel clearly outperform Google's Similar Pages. We have tried to gracefully bring together textual and link information in a common framework where one can compensate for the shortcomings of the other. The Guess Jeans example presented above demonstrates that our scores can leverage link information to handle ambiguous keywords in a manner better than Google can.

The main contribution of this paper, in our view, is the location of our thinking on how to relate

\footnotetext{
${ }^{4}$ WordNet 3.0 contains 155,287 distinct strings.
} 
pages in the context of user intent. As part of our future research agenda we want to formulate relationships between pages that can service user intent outside the domain of information-gathering. We also want to test the applicability of our methods in social networking situations and user-generated content scenarios.

\section{References}

[1] Altavista. http://www.altavista.com/.

[2] A. Aula, N. Jhaveri, and M. Käki. Information search and re-access strategies of experienced web users. In Proc. of 14th Intl. World Wide Web Conference (WWW 2005), 2005.

[3] N. J. Belkin, R. N. Oddy, and H. M. Brooks. ASK for information retreival: Part I. Background and theory. Journal of Documentation, 38(2):61-71, 1982.

[4] N. J. Belkin, R. N. Oddy, and H. M. Brooks. ASK for information retreival: Part II. Results of a design study. Journal of Documentation, 38(3):145-164, 1982.

[5] S. Brin and L. Page. The anatomy of a large-scale hypertextual Web search engine. Computer Networks and ISDN Systems, pages 107-117, 1998.

[6] A. Z. Broder. A taxonomy of Web search. In ACM SIGIR Forum, pages 3-10, 2002.

[7] S. Chakrabarti, B. Dom, P. Raghavan, S. Rajagopalan, D. Gibson, and J. Kleinberg. Automatic resource compilation by analyzing hyperlink structure and associated text. In Proc. 7th Intl. Conference on the World Wide Web (WWW'98), pages 65-74, 1998.

[8] V. Cothey. A longitudinal study of World Wide Web users' information-searching behavior. Journal of the American Society for Information Science and Technology, 53(2):67-78, 2002.

[9] M. de Kunder. The size of the World Wide Web. http://www.worldwidewebsize.com/, Retrieved on 29th February 2008.

[10] J. Dean and M. Henzinger. Finding related pages in the World Wide Web. In Proceedings of the 8th WWW Conference, pages 1467-147, 1999.

[11] C. Fellbaum, editor. Wordnet: An electronic lexical database. Bradford Books, 1998.

[12] A. J. Ferrari, D. Gourley, K. Johnson, F. C. Knabe, D. Tunkelang, and J. S. Walter. Hierarchical data-driven navigation system and method for information retrieval. U.S. Patent number 7,035,864, April 2006.

[13] T. H. Haveliwala, A. Gionis, D. Klein, and P. Indyk. Evaluating strategies for similarity search on the Web. In Proc. 11th Intl. Conference on the World Wide Web (WWW 2002), pages 157-163, 2002 .

[14] S.-H. S. Huang, C. H. Molina-Rodriguez, J. U. Quevedo-Torrero, and M. F. Fonseca-Lozada. Exploring similarity among Web pages using the hyperlink structure. In Proc. International Conference on Information Technology: Coding and Computing (ITCC'04), pages 344-348, 2004.

[15] B. Jansen, D. Booth, and A. Spink. Determining the user intent of Web search engine queries. In Proceedings of the 16th International Conference on World Wide Web, pages 1149-1150, 2007. 
[16] G. Jeh and J. Widom. Simrank: A measure of structural-context similarity. In Proceedings of the 8th ACM SIGKDD International Conference on Knowledge Discovery and Data Mining, 2002.

[17] M. Kellar. An Examination of User Behaviour during Web Information Tasks. PhD thesis, Dalhousie University, Halifax, Canada, 2007.

[18] J. Kleinberg. Authoritative sources in a hyperlinked environment. In Proceedings of the 9th Annual ACM-SIAM Symposium on Discrete Algorithms, pages 668-677, 1998.

[19] S. Lawrence and C. L. Giles. Accessibility of information on the Web. Nature, 400:107-109, 1999.

[20] T.-P. Liang, H.-J. Lai, and Y.-C. Ku. Personalized content recommendation and user satisfaction: Theoretical synthesis and empirical findings. Journal of Management Information Systems, 23(3):45-70, 2006-7.

[21] Z. Lin, M. Lyu, and I. King. Pagesim: A novel link-based measure of Web page similarity. In Proceedings of the 15th International Conference on World Wide Web, pages 1019-1020, 2006.

[22] W. Lu, J. C. M. Janssen, E. E. Milios, N. Japkowicz, and Y. Zhang. Node similarity in the citation graph. Knowl. Inf. Syst., 11(1):105-129, 2007.

[23] W. Lu, J. C. MJanssen, E. E. Milios, and N. Japkowicz. Node similarity in networked information spaces. In Proc. Conference of the Centre for Advanced Studies on Collaborative Research (CASCON), 2001.

[24] G. Marchionini. Information Seeking in Electronic Environments. Cambridge University Press, 1995.

[25] Nutch. http://lucene.apache.org/nutch/.

[26] J. Pitkow and P. Pirolli. Life, death, and lawfulness on the electronic frontier. In Proceedings of ACM SIGCHI Conference on Human Factors in Computing, 1997.

[27] D. E. Rose. Reconciling information-seeking behavior with search user interfaces for the Web. Journal of the American Society for Information Science and Technology, 57(6):797-799, 2006.

[28] D. E. Rose and D. Levinson. Understanding user goals in Web search. In Proc. of 13th Intl. Conference on World Wide Web (WWW 2004), pages 13-19, 2004.

[29] A. Spink, D. Wolfram, M. B. Jansen, and T. Saracevic. Searching the Web: The public and their queries. Journal of the American Society for Information Science and Technology, 52(3):226-234, 2001.

[30] A. Tombros and Z. Ali. Factors affecting Web page similarity. In 27th European Conference on Information Retrieval (ECIR), 2005.

[31] W. Xi, E. Fox, W. Fan, B. Zhang, Z. Chen, J. Yan, and D. Zhuang. Simfusion: Measuring similarity using unified relationship matrix. In Proceedings of the 28th ACM SIGIR Conference on Research and Development in Information Retrieval, pages 130-137, 2005.

[32] Yahoo! Content analysis web services: Term extraction. http://developer.yahoo.com/search/content/V1/termExtraction.html. 